\title{
Valuación financiera de proyectos de inversión en nuevas tecnologías con opciones reales
}

\section{Francisco Antonio}

Álvarez Echeverría

Posgrado de Ingeniería, Facultad de Ingeniería, Universidad Nacional Autónoma de México franlve@yahoo.com.mx

\section{Pablo López Sarabia} División de Estudios de Posgrado, Facultad de Contaduría y Administración, Universidad Nacional Autónoma de México plsarabia@gmail.com

\section{Francisco Venegas-}

\section{Martinez}

Escuela Superior de Economía, Instituto Politécnico Nacional fvenegas1111@yahoo.com.mx

\section{Resumen}

La valuación financiera de proyectos de inversión que involucran nuevas tecnologías requiere de cierto grado de flexibilidad en la implementación de estrategias futuras de negocio como son la expansión, contracción, posposición y abandono del proyecto; asimismo, incorporar la creciente incertidumbre que presentan las nuevas tecnologías a través de tasas de descuento que tengan una dinámica estocástica y que pueden ser simuladas con diversos modelos de tasas de interés como el de Vasicek y CIR que permiten hacer escenarios alternos para evaluar un proyecto de inversión. En este trabajo se desarrolla y aplica la metodología de opciones reales para la adopción de una tecnología del tipo Wi-Fi; para ello se considera una opción real de expansión con el objeto de estimar los flujos de efectivo esperados, así como la rentabilidad del proyecto dentro de un intervalo de tiempo determinado en comparación con la técnica tradicional del valor presente neto (VPN). La tasa de descuento utilizada en la valuación se desprende de la estructura de plazos estimada mediante los modelos Vasicek (1977) y CIR (1985). Los resultados de la valuación reflejan que desde el tercer periodo los flujos de efectivo con opciones reales son positivos, mientras que el VPN lo hace hasta el séptimo periodo, situación que incrementa la probabilidad de tener un VPN negativo que rechace el proyecto, a pesar de que éste tiene viabilidad financiera, si incorporamos el valor de la flexibilidad en el proyecto, el cual no es cuantificado por las técnicas tradicional como el VPN; por lo tanto, se debe complementar el análisis incluyendo una opción real.

Palabras clave: opciones reales, nuevas tecnologías, valuación de proyectos, valor presente neto.

Clasificación JEL: G1, G13, G31 y O32. 


\title{
Financial valuation of projects of new technologies by using real options
}

\begin{abstract}
The financial valuation of investment projects of new technologies requires of a certain degree of flexibility in implementing future investment strategies. Unfortunately, the criterion of net present value is quite rigid in dealing with investment plans because they can not be changed, that is, investment is irreversible. The value of a project on a new technology should not only discount expected flows, but also incorporate flexibility in the future investment strategies such as: expansion, contraction, postponement, abandonment, etc. This paper develops and applies the methodology of real options for the valuation of projects to adopt new technologies. The term structure for discounting the expected flows is estimated by using the Vasicek (1977) and CIR models (1985). The value of the option of adoption is calculated by means of the Black-Scholes formula (1973).
\end{abstract}

Keywords: real options, new technologies, valuation of projects and net present value.

JEL Clasification: G1, G13, G31 and O32.

\section{Introducción}

El ciclo de vida de las nuevas tecnologías es un proceso recursivo que tiende a la mejora continua e innovación de manera permanente, por lo que resulta difícil su exacta ubicación en el tiempo-espacio. Predecir en qué etapa del ciclo surge o desaparece una tecnología en cuestión resulta extremadamente difícil, ya que cada vez los ciclos tecnológicos tienden a acortarse (George y Jones, 2006) debido a diversos factores como los rendimientos, la integración de nuevos competidores, entre otros (Von-Braun y Friedrich, 1991). Por lo anterior, en la actualidad es indispensable contar con una proyección efectiva del capital de trabajo para la sobrevivencia de las corporaciones que dependen del desarrollo tecnológico. La adopción de una nueva tecnología requiere de una adecuada planeación en su fase de implementación, así como de proyecciones confiables de los posibles beneficios - tangibles e intangibles - que se puedan generar, así como de su viabilidad financiera. Los métodos tradicionales de presupuesto de capital y evaluación de proyectos tienen por objeto determinar la viabilidad financiera de un proyecto al determinar la riqueza adicional que genera el proyecto o su rentabilidad, así como el tiempo de recuperación de la inversión. 
Sin embargo, la flexibilidad que pueden tener las nuevas tecnologías y los altos costos de entrada no necesariamente se ven reflejados en las técnicas tradicionales, por lo que pueden resultar inoperantes dentro del campo de la investigación y el desarrollo (R\&D, por sus siglas en inglés) porque dichos métodos sólo pueden ser utilizados para la evaluación de proyectos de corto plazo en mercados con una relativa certidumbre en el largo plazo y que, además, no presente posibilidades de abandono, expansión parcial y cambios en el proyecto que lo hacen más riesgoso para determinar los flujos de efectivo que se tendrán a lo largo del tiempo, así como la tasa de interés más adecuada para descontar dichos flujos de efectivo.

Es claro que la adopción de nuevas tecnologías tiene un cierto grado de riesgo e incertidumbre, por lo que es indispensable realizar una distinción entre la incertidumbre y el riesgo. La incertidumbre es una situación que no permite tener acciones y objetivos bien definidos; por lo tanto, no puede ser administrada, pero sí a menudo puede ser re-conceptualizada en forma de riesgo, el cual es susceptible de ser cuantificable y, en consecuencia, administrable. La cuantificación del riesgo involucrado en la adopción de una nueva tecnología se expresa, generalmente, en una función de la probabilidad, la cual se encuentra inmersa dentro del campo de las expectativas financieras y de negocios. Moenart y Souder (1990) mencionan que dentro de la innovación tecnológica es posible distinguir cuatro fuentes de incertidumbre, a saber:

- Incertidumbre de los consumidores: se refiere a los requerimientos no identificados de los usuarios.

- Incertidumbre tecnológica: se refiere a la falta de conocimientos acerca de soluciones tecnológicas.

- Incertidumbre competitiva: es la ausencia de información acerca de los competidores.

- Incertidumbre de los recursos: se relaciona con la ausencia de información necesaria para crear innovaciones.

La incertidumbre involucrada en los proyectos de nuevas tecnologías generalmente es mayor en la fase inicial, cuando los costos son relativamente bajos, la cual a su vez presenta un nivel de riesgo asociado con cierto nivel de madurez de la tecnología y, consecuentemente, con el proceso de selección de ésta. Para una evaluación correcta y adecuada del riesgo en la adopción tecnológica, entre diversas alternativas, se deben identificar los siguientes factores: 
- Las áreas de incertidumbre asociadas con dicha tecnología.

- El nivel de incertidumbre en cada alternativa.

- El número de enfoques potenciales para el manejo de la incertidumbre (a mayor número menor será el riesgo).

- El riesgo de aplicación de dicha tecnología en cuanto al tiempo de asimilación.

- Los riesgos posventa generados (en cuestión a la capacidad de servicio tecnológico).

- El riesgo de abandono (incapacidad de resolver problemas de manera adecuada).

En consecuencia, en el campo de la investigación y el desarrollo resulta imprescindible utilizar métodos de proyección que permitan cierta flexibilidad en el manejo futuro de las inversiones. Es por ello que en la valuación de proyectos con nuevas tecnologías es insuficiente considerar simplemente el descuento de los flujos de efectivo, pues se debe tener en cuenta las diferentes políticas estratégicas de inversión, así como el desarrollo de futuras oportunidades como la expansión de la inversión, la contracción de la producción, el abandono del proyecto, entre otras, las cuales a final de cuentas resultan ser meramente opciones dentro de la estrategia corporativa. La solución para incorporar dicha incertidumbre y flexibilidad en el proyecto de inversión se encuentra en el uso de las opciones reales, técnica que incorpora los conceptos de las opciones financieras para darle a las técnicas tradicionales la posibilidad de captar los elementos especiales que tienen los proyectos tecnológicos, mineros y petroleros que es donde más se han utilizado. Así, las opciones reales pueden ser complementarias a las técnicas tradicionales de evaluación de proyectos.

Uno de los conceptos fundamentales en las finanzas es el valor del dinero (inversión) a través el tiempo. De hecho, todas las fórmulas teóricas de valuación de activos utilizan este concepto, explícita o implícitamente. Si hoy, al tiempo $t$, se cuenta con $M_{t}$ unidades monetarias, las posibilidades de inversión resultan diversas.

Entre ellas, puede elegirse depositarlo en una institución bancaria para su ahorro. En este último caso, la institución bancaria regresaría posteriormente en un tiempo $T$ el principal, $M_{t}$, más una cantidad adicional, $\Delta M_{t}>0$, conocida como interés. Si se supone que la tasa de interés que retribuye la institución bancaria es constante e igual a $r$ y $M_{0}$ es el depósito inicial, entonces el interés en un periodo anual sería 
$M_{0}=r M_{0}$ y el retorno de la inversión, $M_{t}$, puede ser calculado a través de diversas formas dependiendo del tipo de interés de que se trate. Valencia y Gándara (2009) mencionan que existen diversas técnicas para analizar la viabilidad de los proyectos de inversión, las cuales según Brennan y Trigeorgis (1999) se pueden clasificar como sigue: a) los modelos mecánicos, como el periodo de recuperación, la tasa interna de retorno (TIR) y el valor presente neto (VPN); b) los modelos en donde los flujos son parcialmente controlables y la toma de decisiones responde a la incertidumbre sobre las condiciones exógenas que los afectan; y c) Los modelos dinámicos o de teoría de juegos, en donde no sólo las condiciones exógenas afectan la decisión, sino también las reacciones de otros agentes económicos.

Mediante el criterio de inversión del valor presente neto (VPN) un proyecto nuevo o una nueva estrategia se acepta o se rechaza hoy si VPN $>0$ ó VPN $<0$, respectivamente. Una vez que un proyecto es aceptado bajo este criterio, los planes de inversión no se modifican, es decir, la inversión es irreversible. Por otro lado, desde el criterio del VPN resulta difícil evaluar la opcionalidad de valorar si el entorno de negocios y el ambiente económico son favorables dentro de un cierto número de años, o si un proyecto o estrategia pueda expandirse, simplemente porque hoy no se sabe si en el futuro puedan existir las condiciones para tomar tal decisión (Dixit y Pindick, 2004).

Durante el proceso de presupuestación de las empresas, los miembros de los consejos administrativos toman decisiones que comprometen tanto los recursos como las políticas de desarrollo de la empresa; en dichas decisiones se considera no sólo el capital de trabajo, sino también el desempeño de la organización. En las formas tradicionales de proyección de capital se utiliza el VPN, los flujos de efectivo descontados (FED), la TIR, la relación costo/benefico (C/B), entre otros. En la actualidad, existen muchas empresas que utilizan prácticas y sistemas sofisticados de proyección presupuestaria, en donde el método de FED ha dejado de ser exclusivo para el cálculo y análisis de la incertidumbre financiera (Miller y Waller, 2003; Chatterjee et al., 2003) porque en realidad dicho análisis resulta no ser adecuado bajo ciertas condiciones de mercado específicas (Duku-Kaakyire y Nanang, 2004). Asimismo, en el método VPN subyace la idea de que un proyecto es factible si la empresa puede explotar una ventaja competitiva temporalmente en ausencia de arbitraje (Lander y Pinches, 1998). La adopción de una nueva tecnología requiere de proyecciones confiables de los posibles beneficios. Aunque el método VPN resulta ser una herramienta valiosa en la práctica, requiere que los beneficios estratégicos sean identificados y cuantificados al inicio de cada etapa de inversión, lo cual, en 
ocasiones, puede dejar de lado diversas opciones posibles latentes cuando el riesgo de mercado y la inversión resultan ser activos estratégicos a lo largo de un periodo $T$ determinado (MacDougall y Pike, 2003).

Análogamente, el comportamiento de un proyecto de inversión es muy similar al de las opciones financieras, pues el inversionista tiene la opción de tomar una decisión inmediatamente o esperar a que se revele nueva información; el valor de la opción es el costo de oportunidad de dicho inversionista, que es muy sensible a la incertidumbre sobre el valor futuro del proyecto, por lo que las condiciones cambiantes que lo afectan pueden modificar la decisión de inversión al día de hoy (Valencia y Gándara, 2009). En el horizonte de las opciones financieras, un bono cupón cero (como podría considerarse una opción real, las cuales se revisarán más adelante) es una promesa impersonalizada de pago en el futuro. La cantidad que será pagada recibe el nombre de nominal o principal. Esta promesa se coloca en el mercado a diferentes plazos y, en la fecha de vencimiento, dicho nominal se entrega en una sola emisión. La promesa de pago puede comprarse en el presente con un descuento sobre el valor nominal (Venegas-Martínez, 2006). Muchos de los modelos disponibles para valuar bonos cupón cero se concentran en la evolución de la tasa de interés instantánea, también llamada tasa spot o simplemente tasa corta. El objetivo que persiguen los modelos de tasa corta es explicar en términos estadísticos su comportamiento en el mercado. Así, estos modelos intentan describir esencialmente las propiedades estadísticas del mercado como, por ejemplo, tendencia, reversión, sesgo, curtosis, colas pesadas, intervalos de confianza, probabilidades de ocurrencia, precios promedio, por mencionar algunas (VenegasMartínez, 2006).

Es así que podemos definir la tasa corta como la tasa de interés de plazo más corto disponible en el mercado asociada a un bono cupón cero. Esta tasa no tiene por qué ser la misma que el día siguiente o la semana próxima, y su nivel dependerá de la oferta y la demanda por títulos de deuda al plazo más corto disponible en el mercado (Venegas-Martínez, 2006). En vista de lo anterior, no es posible predecir el comportamiento de dicha tasa, por lo que resulta factible modelarla de forma estocástica a través del movimiento browniano, que no solamente describe las fluctuaciones propias del mercado, sino también proporciona un conjunto de herramientas para su análisis, como sucede con los modelos de tasas de interés Cox, Ingersoll y Ross conocido como CIR (1985) y Vasicek (1977), utilizados en el presente trabajo, para estimar la estructura temporal de tasas de interés incorporando mayor incertidumbre en la tasa con relación al esquema tradicional de 
suponer una tasa libre de riesgo que permita valuar el proyecto de inversión con nuevas tecnologías.

En el área del análisis de las finanzas, las opciones reales tienen su origen en el año de 1977, cuando Meyers propuso el término de opciones reales para valorar proyectos tangibles a través de técnicas de valoración de opciones financieras apoyándose en la similitud que existe entre comprar una opción financiera, obtener recursos, así como las opciones de crecimiento que éstas proporcionan a la empresa (Tamayo, 2006).

En este contexto, Bachini et al. (2007) plantean que:

La característica principal de las opciones reales es que permiten valuar la flexibilidad de las inversiones y las decisiones gerenciales durante el desarrollo de la inversión. A lo largo de la vida de un proyecto de inversión la empresa tiene la posibilidad de tomar decisiones para adecuarse a las distintas situaciones que se presentan y de esta manera aumentar las ganancias esperadas de la inversión o reducir posibles pérdidas. Básicamente, la Teoría de Opciones Reales es una herramienta para valuar estas oportunidades, ampliando el análisis de los proyectos de inversión [...]

De igual manera, estos mismos autores mencionan que el valor de la flexibilidad —que en algunos casos es más importante que el valor del proyecto en sí- es un valor agregado a los proyectos de inversión. La teoría de opciones reales se utiliza para obtener este valor y de esta manera incrementar el valor que posee un proyecto de inversión. En otras palabras, una inversión tiene dos valores distintos: uno sin considerar las opciones reales y otro que incorpora el valor de las opciones reales (Bachini et al., 2007). En este sentido, existe una diferencia entre dos conceptos de valor presente: por un lado, el VPN pasivo es valor actual neto tradicional, es decir, el valor actual de los flujos de fondos futuros de la inversión menos su costo; por otro, el VPN activo que es igual al valor presente neto pasivo más el valor que surge de las opciones reales que pueda contener el proyecto de inversión. Para calcular el valor de las opciones reales se utilizan las técnicas de valuación de opciones financieras debido a las similitudes existentes. Una de las principales técnicas utilizadas en finanzas es la fórmula desarrollada por Black y Scholes (1973) para la valuación de opciones de compra. Para la valuación de opciones reales, como primera aproximación, se utiliza esta misma fórmula, pero cambiando algunas de las variables del modelo (Bachini et al., 2007). 
La diferencia principal es que el activo que se está valuando es un activo real (empresa, proyecto, entre otros) y no un activo financiero. Una de las características que debe presentar un proyecto de inversión para que se pueda aplicar esta teoría es que el proyecto se pueda realizar en cualquier momento del tiempo. Por lo tanto, la pregunta que se hace la empresa no sólo es si debe realizar la inversión o no, sino también cuándo realizarla y en qué escala. La regla de decisión que surge a partir de esta teoría es que la empresa debe invertir si el valor actual neto de la inversión es lo suficientemente grande como para compensar el valor de la opción. Las opciones financieras pueden usarse libre e independientemente, mientras que las opciones reales se utilizan para tomar decisiones estratégicas dentro de la empresa, donde la decisión de tomar una opción debe considerar a la empresa como un todo sistémico (Bachini et al., 2007).

Desde este contexto, las opciones reales son un subconjunto de opciones estratégicas en las que la decisión de ejercicio está fuertemente determinada por el riesgo valorado por los precios del mercado (Sarmiento et al., 2005). Los activos que exhiben un riesgo valorado a precios del mercado están asociados a un conjunto de oportunidades relativamente amplias, en comparación a activos sujetos a riesgos probados (que no se pueden medir a través de las fluctuaciones de los precios de títulos de valores) que permiten, reducir, adquirir o volver a evaluar el riesgo a través de una posición en títulos de valores comerciales. Esto significa que cuando el riesgo es privado, el análisis de las opciones reales es viable cuando el riesgo subyacente puede ser valorado en términos del mercado; de igual forma, este análisis no ofrece alternativas novedosas distintas a las que pueden brindar otros enfoques tradicionales (Sarmiento et al., 2005).

Las opciones reales adquieren una importancia invaluable cuando el grado de incertidumbre es alto y se posee la flexibilidad necesaria para responder a la incertidumbre, y el valor de las opciones reales respecto al VPN es alto, es cercano a cero o negativo (Sarmiento et al., 2005). Lo anterior conlleva a manifestar que el VPN es un caso especial de opción real que se utiliza cuando no se considera la incertidumbre en el proceso de la toma de decisiones y cuyo resultado es la viabilidad o inviabilidad de la decisión.

Mientras el VPN indica la viabilidad del proyecto en un intervalo discreto y temporal, las opciones reales proporcionan alternativas flexibles de manera estocástica, que no exigen la realización inmediata o la cancelación del proyecto como únicas 
alternativas, permitiendo la identificación de una gama más amplia de posibles cursos de acción a través del tiempo (Sarmiento et al., 2005).

Las organizaciones, donde la actividad primordial es exclusivamente la investigación y el desarrollo de nuevas tecnologías, enfrentan problemas muy complejos dentro de la valuación de los proyectos de investigación e innovación tecnológica. Dichos proyectos de investigación y desarrollo (R\&D) se caracterizan por estar inmersos en horizontes de planeación de largo plazo con un alto grado de riesgo e incertidumbre, en donde el valor de la flexibilidad de valuación y factibilidad puede ser vital (Pennings y Lint, 1997). Dentro de este marco, desde hace algún tiempo se han venido utilizando herramientas financieras que permiten establecer cierta flexibilidad en los planes de inversión mediante el uso de un horizonte más amplio que los métodos actuales. De esta forma, Brealey y Myers (1991) señalan que las opciones reales permiten agregar cierto valor a la empresa a través de la amplificación de los bienes de capital o mitigando las pérdidas en los mercados de operación. Cuando se utilizan las técnicas de valuación de proyectos tecnológicos, éstas no incorporan el riesgo de mercado de la opción para su administración (Lander y Pinches, 1998), puesto que utilizan primordialmente una tasa libre de riesgo. Es por lo anterior que algunos autores consideran que el tiempo óptimo de la adopción tecnológica puede ser representado mediante un modelo de irreversibilidad en la inversión, donde el VPN resulta ser una solución subóptima cuando el riesgo y la incertidumbre juegan un papel estratégico en la ejecución de un proyecto en particular (Doraszelski, 2001). Lo anterior no sólo le permite sobrevivir a la organización, sino que también conlleva al desarrollo de ventajas competitivas — como la velocidad de desarrollo, el desarrollo de productos superiores, el desarrollo de productos sin sustitutos definidos, entre otras- debido a que la valuación de la factibilidad de implementación de la nueva tecnología permitiría saber a priori si ésta será redituable y el tiempo en que podría implementarse en un determinado mercado meta mediante la valuación de proyectos de inversión a corto, mediano y largo plazo.

Por lo anterior, el objetivo de este trabajo es incorporar las técnicas de las opciones reales en la valuación de las nuevas tecnologías como un esquema complementario a las herramientas tradicionales de valuación de proyectos, pero incorporando la flexibilidad estratégica que se refleja al incorporar opciones financieras, al tiempo que el riesgo de mercado se refleja a través de una tasa de interés que no sea libre de riesgo y que puede ser estimada mediante diversos modelos de tasas de interés como es el caso de Vasicek. 
La organización del trabajo es como sigue: en la siguiente sección se realiza una revisión sobre las variables y los factores que inciden en la adopción de una nueva tecnología en una organización. Posteriormente, se discute sobre las similitudes de las opciones financieras y las opciones reales y se clasifican los diferentes tipos de opciones reales. Más adelante se aplica la metodología de opciones reales a la valuación financiera de un proyecto de adopción de una nueva tecnología. Por último, se presentan las conclusiones.

\section{El efecto de la irreversibilidad y la indivisibilidad en la adopción de las nuevas tecnologías}

La irreversibilidad y la indivisibilidad han sido el origen de muchas clases conocidas de fallas en los mercados. El traslape de diferentes clases de irreversibilidad e indivisibilidad dentro del cambio estructural generan un proceso dinámico cuyos efectos son evidenciados solamente a través del tiempo con importantes consecuencias en términos de equilibrio y múltiples discontinuidades. La transición de las condiciones del no-equilibrio hacia el equilibrio puede ser obstaculizada o retrasada en forma indefinida debido a estos factores.

El término de irreversibilidad es familiar en la economía industrial, el cual puede ser definido como la dificultad de cambio de un comportamiento o elección dada. Lo anterior puede ser evaluado mediante el costo de oportunidad en un momento dado, $t+1$, desde cualquier intento de compromiso de cambio de un comportamiento o elección dada en un tiempo $t$. Una gran variedad de fenómenos pueden ser catalogados como irreversibilidad, incluyendo los costos asociados entre los productores y los consumidores (switching costs), cuando el cambio de la mezcla de productos o factores de producción entran en juego dentro del paradigma actual, así como también la caída o hundimiento de dichos costos; es decir, la diferencia entre el valor de los activos en el mercado ex-ante y el precio de compra ex-post (Antonelli, 1999).

La indivisibilidad entre los factores de producción conlleva a una gran variedad de fenómenos dentro de las economías técnicas y pecuniarias a gran escala. La importancia de la indivisibilidad y la irreversibilidad se hace presente de una forma fehaciente cuando el análisis económico se focaliza en el rol de la información como un bien económico. La reputación, asimismo, es el resultado de la irreversibilidad y del impacto de la información. 
Los costos de transacción son, de la misma manera, el resultado de una forma especial de indivisibilidad. Bajos niveles de apropiabilidad y aprendizaje pueden describir un caso especial de indivisibilidad. En alusión a lo anterior, la inapropiabilidad cobra importantes implicaciones dinámicas en términos de interdependencia entre los innovadores-usuarios-productores en los sistemas de innovación, así como en los distritos tecnológicos y zonas industriales que promueven la oportunidad de imitación y recombinación tecnológica, lo que da como resultado la obtención de eficiencias terminales mayores en términos de competitividad.

La competitividad y el estándar económico son edificados sobre estrictas asunciones acerca del alcance de las acciones económicas. Los agentes económicos son inducidos a actuar solamente cuando existen procedimientos tendientes a la optimización y a las acciones, las que en este caso resultan ser particular y estrictamente paramétricas; dichas acciones son el ajustamiento de los precios o las cantidades en función del precio. Una acción estructural, como un cambio intencional, es la incorporación de una nueva tecnología en la función de producción o de tendencia en la función de utilidad, lo cual descansa fuera del alcance de los estándares económicos actuales. Los gustos, las tendencias y la tecnología pueden cambiar estrictamente en respuesta de fuerzas endógenas y de la demanda. En estudios recientes sobre inversiones de capital se deja entrever la importancia de la irreversibilidad y las imperfecciones del mercado, y sobre todo las restricciones financieras en dichas inversiones representado éstas un punto fundamental dentro del estudio y desarrollo en el área de la valuación de activos financieros (Holt, 2003).

Por lo anteriormente expuesto, cuando el crecimiento económico es una vía o trayectoria de dependencia, la teoría utilizada en los estados estacionarios resulta ser un marco de referencia inadecuado e inoperante ante tales circunstancias. Los agentes son expuestos a cambios que afectan de manera sensible su comportamiento y sus expectativas; el proceso dentro de las trayectorias de dependencia debería ser considerado como un proceso en el que los agentes no necesariamente tienen un total entendimiento o no poseen la claridad suficiente de la secuencia y el tiempo de duración de cada etapa a través del tiempo. El tiempo, entonces, pasa a ser una fuente de incertidumbre sobre las consecuencias de cada acción implementada o en vías de implementación. Cuando la trayectoria de dependencia es un trabajo, el tiempo resulta ser un factor irreversible: la secuencia de las etapas de crecimiento o desarrollo no pueden, por ninguna circunstancia, retroceder el tiempo y cambiar los perfiles y las intenciones de las acciones, así como tampoco el de sus respectivos efectos. 
Como se mencionó con anterioridad, el tiempo resulta ser un asunto de vital importancia porque afecta en gran medida el proceso de selección; asimismo cabe señalar que la tendencia hacia las condiciones de equilibrio se ve dramáticamente influida por las condiciones y acciones dentro de los mercados, ya que el resultado de cualquier proceso de ajuste dentro de la dinámica de las fuerzas del mercado depende de las condiciones iniciales de éste y del comportamiento de los agentes involucrados en cualquier punto del tiempo.

Cuando ocurre un crecimiento en las trayectorias de dependencia, los principios utilizados para el análisis dentro de una economía estacionaria resultan ser inoperantes dentro de un mercado que se desarrolla en un ambiente dinámico, donde actúan una gran variedad de agentes y fuerzas que propician un estado inicial de desequilibrio.

\section{La adquisición de tecnología externa y la organización}

La decisión de realizar R\&D o adquirir una nueva tecnología depende del grado del crecimiento del mercado y del progreso tecnológico, según se muestra en la figura 1. Al analizar la matriz de la gráfica antes señalada se pueden encontrar tres situaciones distintas para la selección de alternativas:

a) Progreso tecnológico lento y crecimiento del mercado lento/moderado. En esta situación la opción preferible es la $\mathrm{R} \& \mathrm{D}$ e innovaciones internas. La razón de ello es que si se obtienen resultados exitosos en la adopción, se obtendrá una ventaja temporal del producto o de los procesos de producción de la innovación.

b) Progreso tecnológico rápido y crecimiento del mercado lento. En esta situación los esfuerzos en R\&D y nuevas tecnologías resultan riesgosos debido a que pueden conducir al desarrollo de tecnologías no implementables o tecnologías para las que no existirá mercado porque no existe una necesidad a corto plazo para su adquisición tecnológica por parte de las organizaciones.

c) Progreso tecnológico lento y crecimiento del mercado rápido. Para esta situación no hay tiempo suficiente para el desarrollo de R\&D ni innovaciones internas. La estrategia apropiada en este caso en particular estriba en el licenciamiento de la tecnología por parte de la corporación. 
Figura 1

Criterios de selección de adquisición de tecnología (Abbetti, 1989)

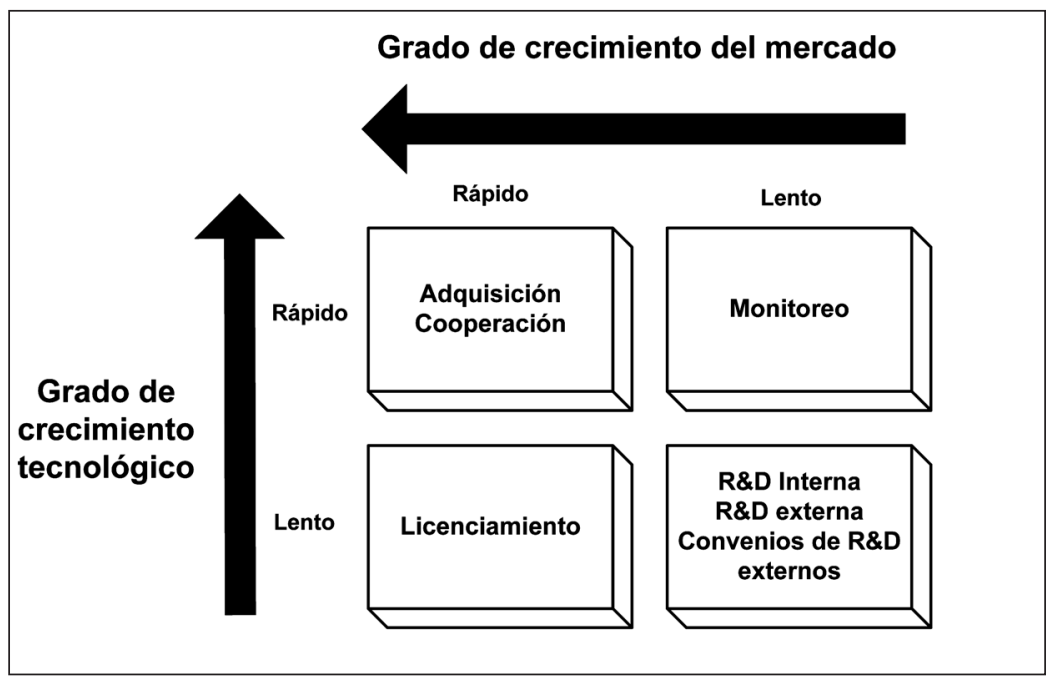

Aunado a lo anterior, y en forma complementaria, para el desarrollo de criterios adecuados que permitan la optimación en la selección y evaluación de alternativas dentro de la adopción de nuevas tecnologías hacia el interior de una organización se deben tener en cuenta los siguientes aspectos (Abetti, 1989):

- El grado de ventaja disponible para la empresa después de la implantación, lo cual depende del grado de autonomía con respecto a la utilización de la tecnología.

- El tiempo de retraso necesario para la incorporación de la tecnología en cuestión, como bienes de la organización.

- El costo de adquisición relativo de la tecnología.

- El riesgo relativo que representa la adquisición tecnológica en lo concerniente a su no incorporación al seno de la corporación u organización.

- Los posibles beneficios que se pudiese obtener de su implementación.

Valuación financiera de estrategias de negocio mediante la utilización de opciones reales

El auge de los mercados derivados en los últimos años se ha basado en la especulación, aunque la esencia de dichos instrumentos como las opciones y futuros 
es la cobertura de riesgos. Precisamente, la posibilidad de separar el riesgo de las fluctuaciones en los precios de las operaciones físicas subyacentes de una empresa y gestionarlos de manera individual, a través del uso de productos derivados, es la mayor de las innovaciones financieras de las últimas décadas; por ello, a medida que se van creando productos con esta finalidad se aprecia su aplicación, no sólo en la cobertura de riesgos, sino también para cubrir otras necesidades de la organización. Así, el punto central que tienen los administradores de proyectos y presupuestos de capital es la utilización de las opciones reales en la valuación de un proyecto de inversión al reconocer que los conceptos de las opciones financieras pueden ser utilizados de manera análoga para la valuación de activos reales que presentan oportunidades estratégicas para diferentes opciones de negocio dentro de la organización.

Sin embargo, las opciones reales utilizadas por las corporaciones suelen ser, generalmente, un poco más complejas que las opciones financieras. Esto ocurre especialmente en áreas en donde existen una gran cantidad de estrategias interrelacionadas a través del tiempo dentro de un mismo proyecto de capital. Es en este contexto en donde las opciones financieras comúnmente utilizadas para la valuación de activos muestran la incapacidad de valorar múltiples opciones dentro de las opciones reales para los proyectos de inversión de capital (Pinches, 1998).

La metodología de valuación financiera de proyectos mediante la utilización de opciones reales se ha convertido en una herramienta potencial para la toma de decisiones en proyectos de inversión o estrategias de negocios cuando existe la flexibilidad (opcionalidad) de tomar en el futuro nuevas decisiones relacionadas con dichos proyectos o estrategias como pueden ser la extensión, la contracción, la posposición, la corrección o el abandono de un proyecto.

La metodología de opciones reales, en términos generales, es la aplicación de las técnicas de valuación de opciones financieras a la valuación de proyectos de inversión y estrategias de negocios cuando existe la flexibilidad de tomar, en el futuro, nuevas decisiones relacionadas con dichos proyectos y estrategias.

\section{Comparativa entre opciones financieras y opciones reales}

De igual forma que en las opciones financieras, el valor de una opción real aumenta con el tiempo de maduración y con la volatilidad subyacente. Lo anterior implica que la flexibilidad de tomar nuevas decisiones en un futuro próximo o lejano 
tiene un valor mayor cuando el horizonte de planeación aumenta y/o cuando hay mayor incertidumbre sobre los resultados esperados. En el cuadro 1 se presenta la correspondencia entre los parámetros de una opción financiera y los de una opción real. De esta manera, una empresa a partir de un tiempo t, posee una oportunidad de invertir en un proyecto subyacente hasta el tiempo $T$, lo cual podría verse como una opción para adquirir un cierto valor presente de los flujos esperados, $S_{T}$, a cambio una inversión, $K$, en la fecha de vencimiento. En este caso, $\mathrm{S}_{\mathrm{T}}$ tiene asociado un factor de incertidumbre, a saber, la volatilidad de los flujos de efectivo del proyecto. En este sentido, la opción solamente será ejercida cuando $S_{T}>K$, en cuyo caso el inversionista permanece en el proyecto subyacente, pues de lo contrario será conveniente abandonarlo. Esto lleva a inferir que mientras las opciones financieras tratan con activos financieros, las opciones reales tratan con activos reales como pueden ser unidades de negocio, obras e infraestructura, nuevas tecnologías, entre otros, generados a través de proyectos de inversión (Venegas-Martínez, 2006).

Las opciones reales, a diferencia de las opciones financieras, no consideran series históricas de tiempo para estimar la volatilidad sobre el activo subyacente. Otra de las diferencias entre las opciones financieras y las reales es que mientras en las primeras el valor del subyacente es conocido en las opciones reales el valor presente puede ser estocástico. Sin embargo, la incertidumbre en la estimación del valor del subyacente no cambiará el valor de la opción en el presente, ni tampoco la regla de inversión en la maduración de la opción. Cuando la opción se encuentra dentro del flujo de efectivo en el tiempo de maduración, la regla es "invertir". Pero cuando el valor del subyacente resulta ser de naturaleza estocástica, la opción podría estar fuera de toda consideración de inversión después de que el producto o el servicio han sido introducidos. No obstante, el valor del subyacente es un estimador neutral, por lo que el valor esperado como resultado del valor del proyecto es igual al valor estimado del subyacente. Es por ello que la regla de inversión en la maduración de la opción depende del valor del subyacente y es irrelevante lo que pudiera ocurrir alrededor de éste (Pennings y Lint, 1997). 


\section{Cuadro 1}

Equivalencias entre las opciones financieras y las opciones reales

\begin{tabular}{l|l|l}
\hline \multicolumn{1}{c|}{ Parámetro } & \multicolumn{1}{c}{ Opción real } & \multicolumn{1}{c}{ Opción financiera } \\
\hline$S_{t}$ & $\begin{array}{l}\text { Valor presente de los flujos esperados } \\
\text { en } t\end{array}$ & Subyacente \\
\hline$K$ & Costo de inversión en $T$ & Precio de ejercicio \\
\hline$R$ & Tasa de interés libre de riesgo & Tasa libre de riesgo \\
\hline$\sigma$ & $\begin{array}{l}\text { Volatilidad de los flujos de efectivo del } \\
\text { proyecto }\end{array}$ & Volatilidad subyacente \\
\hline$T-t$ & $\begin{array}{l}\text { Tiempo en que la oportunidad de } \\
\text { invertir desaparece }\end{array}$ & Plazo del vencimiento \\
\hline
\end{tabular}

Fuente: adaptado de Venegas-Martínez, 2006

\section{Diferentes tipos de opciones reales}

Para la valuación de proyectos de inversión (mediante la consideración de diversas variables y escenarios) se pueden utilizar diversas clases de opciones reales existentes dentro de la literatura (Lander y Pinches, 1998; Venegas-Martínez, 2006) que en algunos casos suponen una complejidad analítica mayor que los métodos clásicos (Trigeorgis, 1991 y 1993; Perlitz et al., 1999). Es importante destacar que en el caso de valuación con opciones reales la tasa de descuento es una tasa libre de riesgo de incumplimiento. Una oportunidad corporativa de inversión se puede valuar como una opción (europea o americana) de compra, pues la empresa obtiene el derecho, mas no la obligación, de llevar a cabo cierta estrategia de inversión. La correspondencia entre las características del proyecto (extensión, contracción, posposición, corrección, abandono, por mencionar algunas) y los parámetros determinan el valor de la opción en cuestión. Entre las diferentes opciones reales que pueden utilizarse para la valuación de proyectos tecnológicos se tienen:

a) Opción real de expansión.

b) Opción real de contracción.

c) Opción real de cierre temporal.

d) Opción real de permanencia.

e) Opción real de abandono.

f) Opción real de cambio tecnológico. 


\section{Valuación de un proyecto de adopción de nuevas tecnologías}

Con el propósito de mostrar las ventajas del uso de las opciones reales versus VPN, se presenta la valuación de un proyecto de una compañía de desarrollo tecnológico que por razones de confidencialidad no se revela su nombre, aunque para fines del artículo no es indispensable, ya que lo que se desea es ilustrar el potencial de las opciones reales para valuar nuevas tecnologías que pueden presentar oportunidades de negocios y alto riesgo e incertidumbre.

La empresa analizada desea realizar el cambio y/o incorporación de una nueva tecnología con la finalidad de expandir su mercado y proveer un mejor servicio a sus clientes. En primera instancia, se requiere la valuación financiera del proyecto con el objeto de observar su rentabilidad en un horizonte de tiempo no muy lejano, debido al dinamismo que envuelve al mercado en cuestión. La tecnología que se pretende incorporar dentro de la empresa es el sistema de conectividad inalámbrico de Wi-Max (en la actualidad sus operaciones involucran tecnología Wi-Fi). Wi-Max (del inglés Worldwide Interoperability for Microwave Access) es un estándar de transmisión inalámbrica de datos que proporciona accesos concurrentes en áreas de hasta $48 \mathrm{~km}$ de radio y a velocidades de hasta $70 \mathrm{Mbps}$ utilizando tecnología que no requiere visión directa con las estaciones base. Wi-Max es un concepto parecido al Wi-Fi (por sus siglas en inglés, Wireless Fidelity), pero con mayor cobertura y ancho de banda. Wi-Fi fue diseñada para ambientes inalámbricos internos como una alternativa al cableado estructurado de redes y con capacidad sin línea de vista de corto alcance. Wi-Max, por el contrario, fue diseñado como una solución de última milla en redes metropolitanas (WAN) para prestar servicios en el nivel comercial.

Valuación financiera de un proyecto de adopción de nueva tecnología del tipo Wi-Max y Wi-Fi

Para la valuación financiera de un proyecto de adopción tecnológica, se procederá en primera instancia a estimar la estructura de plazos. Es importante recordar que en el caso de valuación con opciones reales, la tasa con la que se descuentan los flujos del proyecto en cuestión tiene que ser una tasa libre de riesgo de incumplimiento. Para la construcción de la estructura de plazos, dentro de la metodología, se incorporaron dos metodologías diferentes con el objeto de obtener un panorama más amplio del comportamiento de las tasas de descuento de los proyectos, considerándola como una variable estocástica. Los dos modelos escogidos para 
la incorporación a la metodología son el modelo de tasa corta de Vasicek (1977) y el modelo de Cox, Ingersoll y Ross (1985). En el modelo de Vasicek (1977) se considera la dinámica de una tasa corta que presenta reversión a la media hacia un valor constante. Este comportamiento se observa en diversos casos cuando se analizan series de tiempo de tasas a corto plazo. Es imprescindible mencionar que la estructura de plazos generada por el modelo de Vasicek (1977) puede producir en ocasiones tasas negativas con probabilidades positivas (cuando $\beta=0$ ). Afortunadamente, esta limitación se desvanece con el modelo propuesto por Cox, Ingersoll y Ross (1985), ya que en ese modelo las tasas siempre son positivas (VenegasMartínez, 2006). Cabe mencionar que para la estimación de la estructura de plazos se utilizó la información de los rendimientos obtenidos por los CETES entre el 22 de marzo de 2007 y el 11 de octubre de 2007.

Construcción de la estructura de plazos mediante el modelo de Vasicek (1977) y CIR (1985)

El modelo de Vasicek genera precios de bonos a diferentes rendimientos, $\mathrm{B}(\mathrm{t}, \mathrm{T})$, $\mathrm{T} \geq \mathrm{t}$. A partir de estos precios se generará la estructura de plazos de la tasa de interés, $\mathrm{R}(\mathrm{t}, \mathrm{T})$, con la siguiente relación:

$$
R(t, T)=-\frac{1}{T-t} \ln B(t, T)
$$

El resultado principal del modelo de Vasicek es que

$$
R(t, T)=r_{t} \frac{1-e^{-a(T-t)}}{a(T-t)}-\left(\frac{1-e^{-a(T-t)}}{1-e^{-a(T-t)}}-1\right)\left(b-\frac{\sigma^{2}}{2 a^{2}}\right)+\frac{\sigma^{2}\left(1-e^{-a(T-t)}\right)}{4 a^{3}(T-t)}
$$

Los parámetros a y b pueden ser estimados utilizando un modelo de regresión lineal simple con el supuesto estándar de errores normales no correlacionados, o bien mediante un proceso autoregresivo de orden uno con tendencia. Para fines prácticos, el modelo de Vasicek puede plantearse en términos discretos como una ecuación estocástica en diferencias. Si se escribe $\beta_{0}=\mathrm{ab}$ y $\beta_{1}=1-\mathrm{a}$, una versión discreta del modelo sería:

$$
r_{t}=\beta_{0}+\beta_{1} r_{t-1}+\varepsilon_{t},
$$


donde $\left\{\varepsilon_{\mathrm{t}}\right\}$ son variables aleatorias independientes y normalmente distribuidas con media cero y variancia $\sigma^{2}$. La media incondicional de $r_{t}$ es igual a:

$$
\mathrm{E}\left[r_{t}\right]=\frac{\beta_{0}}{1-\beta_{1}}=b
$$

y la variancia incondicional está dada por:

$$
\operatorname{Var}\left[r_{t}\right]=\frac{\sigma^{2}}{1-\beta_{1}^{2}}=\frac{\sigma^{2}}{1-(1-a)^{2}}
$$

Una vez realizadas las operaciones matriciales (mediante las ecuaciones de YuleWalker para métodos auto-regresivos) se obtuvieron los siguientes coeficientes:

$$
\left(\begin{array}{l}
\phi_{1} \\
\phi_{2}
\end{array}\right)=\left(\begin{array}{c}
0.018539025 \\
0.74235845
\end{array}\right)
$$

La variancia obtenida fue:

$$
\hat{\sigma_{\xi}^{2}}=\frac{(b-A \hat{\Phi})^{T}(b-A \hat{\Phi})^{T}}{t-2 p}=(2.15021 \mathrm{E}-07)
$$

y la matriz de variancias-covariancias es igual a:

$$
\hat{\sum}_{\Phi_{p}}=\hat{\sigma}_{\xi}^{2}\left(A^{T} A / t\right)^{-1}=\left(\begin{array}{cc}
0.00197707 & -0.02756823 \\
-0.02756823 & 0.384455906
\end{array}\right)
$$

Por lo tanto, $\Phi_{1}(b)=0.018539025$ y $\Phi_{2}(a)=0.74235845$, entonces $\beta_{0}=a b$ y $\beta_{1}=1-a$. Así, la versión discreta del modelo estaría dada por:

$$
r_{t}=0.0720+0.2576 r_{t-1}+\varepsilon_{t}
$$

La estructura de plazos es definida a través de la ecuación (2) con $a=0.0720$, $b=0.2576, r_{t}=0.0717$ y $\sigma=0.0004637$ (los coeficientes estimados son significativamente distintos de cero con un $95 \%$ de confianza), con lo cual se obtiene la curva 
mostrada en la figura 2. La estructura de plazos de Vasicek es creciente y en el largo plazo tiende al valor de $7.16768075 \%$. En el caso del CIR es posible obtener curvas de rendimiento con pendientes positivas, con pendientes negativas o con máximos y/o mínimos. La curva de rendimiento del modelo CIR se calcula como sigue:

$$
\begin{gathered}
R(t, T)=-\frac{\ln B(t, T)}{T-t} \\
R(t, T)=\frac{r_{t} D(t, T)-A(T-t)}{T-t}
\end{gathered}
$$

En donde:

$$
\begin{gathered}
D(t, T)=\frac{2\left(e^{\sqrt{a^{2}+2 \sigma^{2}}(T-t)}-1\right)}{\left(a+\sqrt{a^{2}+2 \sigma^{2}}\right)\left(e^{\sqrt{a^{2}+2 \sigma^{2}(T-t)}}-1\right)+2 \sqrt{a^{2}+2 \sigma^{2}}} \\
D(t, T)=\frac{2 a b}{\sigma^{2}} \ln \left[\frac{2\left(a+\sqrt{a^{2}+2 \sigma^{2}}\right) e^{a+\sqrt{a^{2}+2 \sigma^{2}}(T-t) * 0.5}-1}{\left(a+\sqrt{a^{2}+2 \sigma^{2}}\right)\left(e^{\sqrt{a^{2}+2 \sigma^{2}}(T-t)}-1\right)+2 \sqrt{a^{2}+2 \sigma^{2}}}\right]
\end{gathered}
$$

La ecuación del modelo CIR en versión discreta puede escribirse como sigue:

$$
y_{t+1}=\beta_{1} y_{t}^{-1}+\beta_{2} y_{t}+\varepsilon_{t}
$$

Donde $y_{t}=2 \sqrt{r_{t}}, \beta_{1}=2 a b-\left(\sigma^{2} / 2\right), \beta_{2}=1-(a / 2)$ y $\varepsilon_{t} \approx N\left(0, \sigma^{2}\right)$. Si $\tilde{\beta}_{1}, \tilde{\beta}_{2}, \tilde{\sigma}^{2}$ son estimadores de mínimos cuadrados ordinarios, MCO, los cuales son de máxima verosimilitud, entonces:

$$
\tilde{a}=2\left(1-\tilde{\beta}_{2}\right)
$$

$\mathrm{Y}$

$$
\tilde{\mathrm{b}}=\frac{2 \tilde{\beta}_{1}+\tilde{\sigma}^{2}}{4 \tilde{\mathrm{a}}}=\frac{2 \tilde{\beta}_{1}+\tilde{\sigma}^{2}}{8\left(1-\tilde{\beta}_{2}\right)}
$$


Son estimadores de máxima verosimilitud también. Una vez resuelto el sistema mediante mínimos cuadrados se obtienen los resultados mostrados en el cuadro 2 .

\section{Cuadro 2}

Parámetros estimados para el modelo CIR (MCO)

\begin{tabular}{c|c}
\hline \multicolumn{2}{c}{ Parámetros } \\
\hline Coeficiente $\mathbf{b}_{2}$ & Coeficiente $\mathbf{b}_{1}$ \\
0.88913008 & 3.189626134 \\
\hline Coeficiente $\hat{a}$ & Coeficiente $\hat{b}$ \\
0.221739841 & 7.192611578 \\
\hline Error estándar del coeficiente & Error estándar del coeficiente \\
\hline 0.05379105 & 1.541814315 \\
\hline Coeficiente de determinación & Error estándar \\
\hline 0.99999020 & 0.017376094 \\
\hline Valor observados de $\mathbf{F}$ & Grados de libertad \\
\hline 1377729.19352765 & 27 \\
\hline $\begin{array}{c}\text { Suma para la regresión de los } \\
\text { cuadrados }\end{array}$ & Suma residual de los cuadrados \\
831.95184793 & 0.008152074 \\
\hline
\end{tabular}

Con todo ello procedemos a calcular la estructura de plazos correspondiente mediante la utilización de las fórmulas anteriores; de esta forma, se obtiene la figura 2, en donde puede observarse las estructuras de plazos tanto del CIR como la de Vasicek. Si se observa con cuidado el comportamiento de la estructura de plazos propuestas, cabe la posibilidad de utilizar las dos estructuras de plazo de acuerdo con las expectativas y consideraciones de los inversionistas, es decir, si se utiliza un criterio conservador se utilizaría la de estructura de Vasicek, ya que a través del tiempo se espera que las tasas y los rendimientos sean crecientes y no decrecientes; sin embargo, si se espera un entorno futuro con un decrecimiento de las tasas de referencia debido a un ambiente económico turbulento se utilizaría la estructura de CIR. 
Figura 2

Estructura de plazos a diez años estimada con Vasicek y CIR

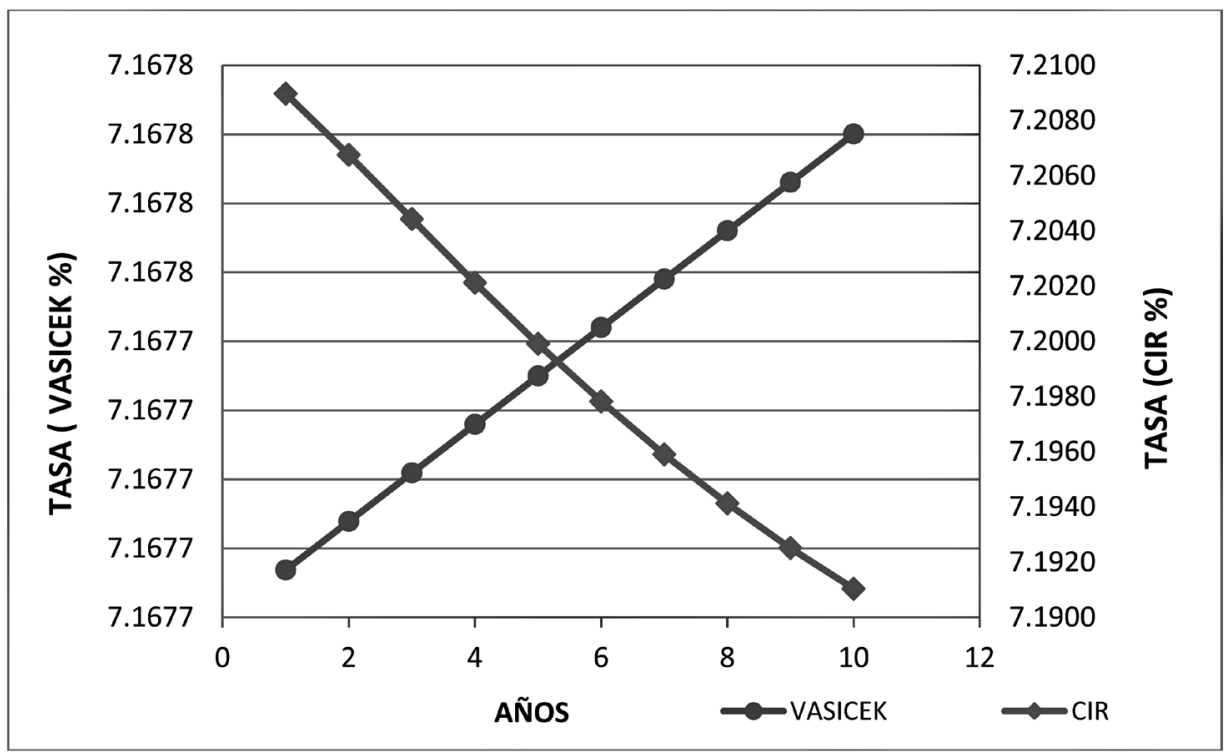

La estructura temporal de tasas de interés es una variable relevante para la valuación del proyecto, ya que en caso de que una curva de la estructura fuera plana o invertida cambiaría la valuación del proyecto, más aún si consideramos que la dinámica de la tasa es estocástica y, por tanto, se utilizan diversos modelos de tasas de interés con el fin de tener más elementos que permitan hacer una evaluación adecuada del proyecto de inversión en un escenario de incertidumbre y flexibilidad en las estrategias de negocios.

\section{Valuación financiera utilizando el modelo de Black-Scholes}

Una vez calculada la estructura de plazos, se procede al cálculo del valor de la opción. Para este caso en particular se utilizará una opción real de expansión (prima de una opción europea de compra, debido a que el proyecto se encuentra por implementarse) para así determinar la factibilidad del proyecto, donde la empresa podría expandir el proyecto en una proporción, $\alpha$, para lo cual se requiere invertir una cantidad adicional $\mathrm{K}^{\prime}$ en un tiempo T. Esta posibilidad estratégica tiene una opción asociada con el proyecto subyacente existente. Si $(1+\alpha) S_{T}-K^{\prime}$ es el valor presente neto aumentado en la proporción $\alpha$ menos el costo de la inversión adicio- 
nal $\mathrm{K}^{\prime}$ al tiempo $\mathrm{T}$, el valor intrínseco (valor en la fecha de vencimiento) de esta opción está dado por:

$$
c_{e}\left(S_{T}, T ; \alpha, K^{\prime}\right)=\max \left((1+\alpha) S_{T}-K^{\prime}, S_{T}\right)
$$

donde $\mathrm{K}=\mathrm{K}^{\prime} / \alpha$. En particular, si el valor presente de los flujos de efectivo esperados es conducido por un movimiento geométrico browniano "neutral al riesgo", es decir:

$$
\mathrm{d} S_{t}=r S_{t} \mathrm{~d} t+\sigma S_{t} \mathrm{~d} W_{t}
$$

Siendo $r$ la tasa de interés libre de riesgo (de incumplimiento) y $\sigma>0$ es la volatilidad instantánea de $S_{t}$, se tiene que el valor de la opción real de expansión en $t$ está dado por:

$$
\begin{gathered}
c\left(S_{t}, t\right)=e^{-r(T-t)} \mathrm{E}\left[S_{T}+\alpha \max \left(S_{T}-K, 0 \mid F_{\mathrm{t}}\right]\right. \\
=e^{-r(T-t)} \mathrm{E}\left[S_{T} \mid S_{t}\right]+\alpha c^{-r(T-t)} \int_{K}^{\infty}(s-K) f_{S_{T} \mid S_{t}}\left(s \mid S_{t}\right) \mathrm{d} s \\
=S_{t}+\alpha c_{\mathrm{BS}}\left(S_{t}, t\right)
\end{gathered}
$$

donde

$$
\begin{gathered}
f_{S_{T} \mid S_{t}}\left(s \mid S_{t}\right)=\left(\frac{1}{\sqrt{2 \pi(T-t) \sigma s}}\right) \exp \left\{-\frac{1}{2}\left(\frac{\ln \left(\frac{s}{S_{t}}\right)-\left(r-\frac{1}{2} \sigma^{2}\right)(T-t)}{\sigma \sqrt{T-t}}\right)^{2}\right\} \\
c_{\mathrm{BS}}\left(S_{t}, t\right)=S_{t} \Phi\left(d_{1}\right)-K e^{-r(T-t)} \Phi\left(d_{2}\right)
\end{gathered}
$$




$$
\begin{gathered}
\Phi(d)=\mathrm{P}_{\varepsilon}(\varepsilon \leq d)=\int_{-\infty}^{d} \frac{1}{\sqrt{2 \pi}} e^{-\frac{1}{2} \varepsilon^{2}} \mathrm{~d} \varepsilon=1-\Phi(-d), \\
d_{1}=\frac{\ln \left(\frac{S_{t}}{K}\right)+\left(r+\frac{1}{2} \sigma^{2}\right)(T-t)}{\sigma \sqrt{T-t}} \\
d_{2}=\frac{\ln \left(\frac{S_{t}}{K}\right)+\left(r-\frac{1}{2} \sigma^{2}\right)(T-t)}{\sigma \sqrt{T-t}}=d_{1}-\sigma \sqrt{T-t}
\end{gathered}
$$

Es decir, $\mathrm{c}_{\mathrm{BS}}\left(\mathrm{S}_{\mathrm{t}}, \mathrm{t}\right)$ es la fórmula de Black-Scholes (1973) para valuar una opción de compra. Es importante destacar que los flujos de efectivo esperados no son un activo que se compre o venda en un mercado definido, lo que genera una situación de mercados incompletos.

Asimismo, cabe recalcar que para la valuación del proyecto se tomaron en cuenta los flujos de efectivo, la utilidad neta esperada, la inversión inicial y las volatilidades de los flujos de efectivo a través del tiempo; todos estos valores fueron retomados a partir del plan de negocios de inversión de una empresa que incorporó una nueva tecnología del tipo Wi-Max y Wi-Fi; dicha empresa fungirá como representativa para nuestro análisis, como ya se había indicado previamente. Lo relevante de este trabajo es la comparación de metodologías de opciones reales y valor presente neto, por lo que es irrelevante el nombre de la empresa y sólo interesa la definición de las condiciones iniciales. Los parámetros involucrados en el cálculo se presentan en el cuadro 3 .

Cuadro 3

Parámetros utilizados para la evaluación del proyecto tecnológico

\begin{tabular}{c|l}
\hline Parámetro & \multicolumn{1}{c}{ Valor } \\
\hline$S_{+}$ & Valor presente de los flujos de efectivo futuros $\left(\$ 2430000.00^{*}\right)$ \\
\hline$K$ & Costo de implementación $\left(\$ 500000.00^{*}\right)$ \\
\hline$r$ & Tasa libre de riesgo $(\%$, estructura de plazos de Vasicek) \\
\hline$T$ & Tiempo de expiración (10 años) \\
\hline$\sigma$ & Volatilidad (20\%) \\
\hline$V P N$ & Valor presente neto de las utilidades (\$400 000.00*) \\
\hline$I_{0}$ & Inversión inicial (\$ 2 000 000.00*) \\
\hline *Todas las cantidades se encuentran expresadas en dólares estadounidenses \\
\hline
\end{tabular}


Una vez determinados los valores de los parámetros, se procede al cálculo de resultados, los cuales se muestran en el cuadro 4. Asimismo, se calculó el valor presente neto (VPN) de las ganancias esperadas, obtenidas del estado de resultados (con fines de comparación); los resultados se muestran en el cuadro 5.

\section{Cuadro 4}

Valor y parámetros de la opción en un horizonte de 10 años

\begin{tabular}{|c|c|c|c|c|}
\hline \multicolumn{5}{|c|}{ VASICEK } \\
\hline$K \mathrm{e}^{-r t}$ & $R(0, T)(\%)$ & 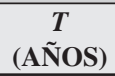 & $c(0, T)$ & $I_{0}-c(0, T)$ \\
\hline $465,415.89$ & 0.071677 & 1 & $1,964,584.11$ & $-35,415.89$ \\
\hline $433,223.96$ & 0.071677 & 2 & $1,996,776.04$ & $-3,223.96$ \\
\hline $403,258.98$ & 0.071676 & 3 & $2,026,741.03$ & $26,741.03$ \\
\hline $375,367.10$ & 0.071676 & 4 & $2,054,633.02$ & $54,633.02$ \\
\hline $349,405.07$ & 0.071675 & 5 & $2,080,595.54$ & $80,595.54$ \\
\hline $325,239.52$ & 0.071674 & 6 & $2,104,762.37$ & $104,762.37$ \\
\hline $302,746.27$ & 0.071673 & 7 & $2,127,257.90$ & $127,257.90$ \\
\hline $281,809.68$ & 0.071672 & 8 & $2,148,197.74$ & $148,197.74$ \\
\hline $262,322.11$ & 0.071671 & 9 & $2,167,689.32$ & $167,689.32$ \\
\hline $244,183.35$ & 0.071669 & 10 & $2,185,832.58$ & $185,832.58$ \\
\hline \multicolumn{5}{|c|}{ CIR } \\
\hline$K \mathbf{e}^{-r t}$ & $R(0, T)(\%)$ & $\begin{array}{c}T \\
\text { (AÑN) } \\
\end{array}$ & $c(0, T)$ & $I_{0}-c(0, T)$ \\
\hline $465,223.71$ & 0.072090 & 1 & $1,964,776.29$ & $-35,223.71$ \\
\hline $432,885.39$ & 0.072068 & 2 & $1,997,114.61$ & $-2,885.39$ \\
\hline $402,814.11$ & 0.072044 & 3 & $2,027,185.89$ & $27,185.89$ \\
\hline $374,848.95$ & 0.072021 & 4 & $2,055,151.16$ & $55,151.16$ \\
\hline $348,839.74$ & 0.071999 & 5 & $2,081,160.86$ & $81,160.86$ \\
\hline $324,647.14$ & 0.071978 & 6 & $2,105,354.72$ & $105,354.72$ \\
\hline $302,141.40$ & 0.071959 & 7 & $2,127,862.69$ & $127,862.69$ \\
\hline $281,203.30$ & 0.071941 & 8 & $2,148,803.99$ & $148,803.99$ \\
\hline $261,721.82$ & 0.071925 & 9 & $2,168,289.42$ & $168,289.42$ \\
\hline $243,594.44$ & 0.071910 & 10 & $2,186,421.22$ & $186,421.22$ \\
\hline
\end{tabular}




\section{Cuadro 5}

\section{VPN en el proyecto de inversión}

\begin{tabular}{c|c|c}
\hline Años & $F_{i}=\frac{F_{0}}{\left(1+r_{i}\right)^{i}}$ & $V P N=-I_{0}+\sum_{i=1}^{n=1} \frac{F_{0}}{\left(1+r_{i}\right)^{i}}$ \\
\hline 1 & $373,273.61$ & $-1,626,726.39$ \\
\hline 2 & $348,332.97$ & $-1,278,393.42$ \\
\hline 3 & $325,058.76$ & $-953,334.66$ \\
\hline 4 & $303,339.64$ & $-649,995.02$ \\
\hline 5 & $283,071.71$ & $-366,923.31$ \\
\hline 6 & $264,158.00$ & $-102,765.31$ \\
\hline $\mathbf{7}$ & $\mathbf{2 4 6 , 5 0 8 . 0 2}$ & $\mathbf{1 4 3 , 7 4 2 . 7 1}$ \\
\hline 8 & $230,037.35$ & $373,780.06$ \\
\hline 9 & $214,667.18$ & $588,447.24$ \\
\hline 10 & $200,323.98$ & $788,771.22$ \\
\hline
\end{tabular}

Si se observa con cuidado la última columna del cuadro 4 y la última columna del cuadro 5, se destaca que mediante el uso de opciones reales, los flujos de efectivo del proyecto comienzan a ser positivos a partir del tercer año (ver figura 3 ), no importando qué tipo de estructura de plazos se utilice (creciente o decreciente); en cambio utilizando un método de proyección lineal, como es el VPN, los flujos de efectivo se hacen positivos hasta el séptimo año (ver figura 3). Si la decisión sobre la inversión es irreversible, mediante el criterio del VPN, el proyecto podría ser rechazado, ya que implicaría trabajar con pérdidas hasta el séptimo año de operación. Si por el contrario cuando se tiene un criterio de VPN extendido $(\overline{V P N})$ de la forma $\overline{V P N}=V P N+c$ (donde c es la opción real), entonces se tiene mayor flexibilidad en la valuación de un proyecto. Dicha flexibilidad u opcionalidad, de tomar en un futuro una nueva decisión de inversión posee un valor presente $c$; es decir, $\overline{V P N}=V P N+c$, entonces aunque $\mathrm{VPN}<0$, si $\overline{V P N}=V P N+c>0$, la viabilidad de extender o implementar el proyecto o estrategia es viable. Por lo tanto, el proyecto bajo este criterio resulta viable en su realización, pues de acuerdo con los resultados obtenidos, el proyecto empezaría a ser rentable a partir del tercer año (ver comparativa en figura 3 ). 
Figura 3

Comparativa de los flujos esperados* del proyecto tecnológico evaluado a partir de dos metodologías diferentes: VPN y opciones reales (*Unidades en dólares estadounidenses)

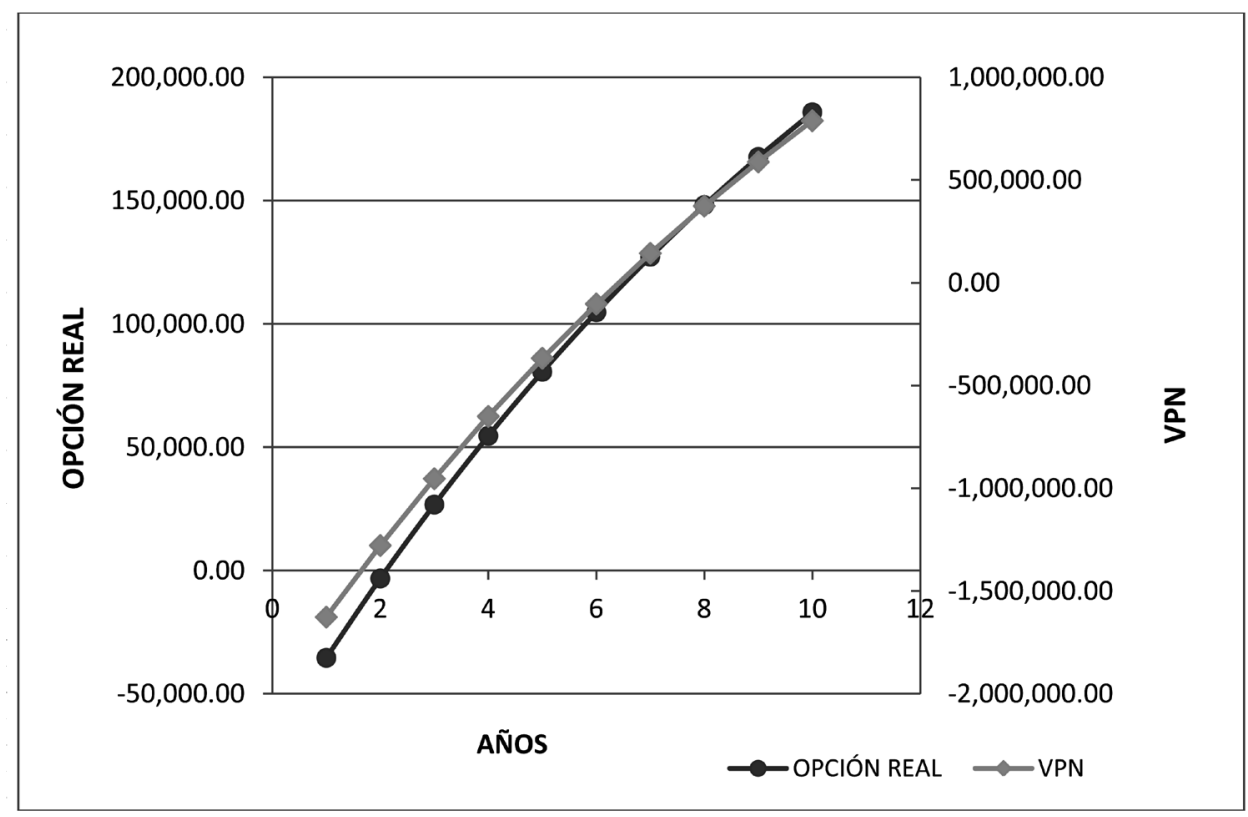

\section{Conclusiones}

Las opciones reales, en general, pueden mejorar la capacidad del proceso de toma de decisiones concernientes a la inversión de activos por las siguientes razones: 1) representan una herramienta flexible para la toma de decisiones; 2) proporcionan modelos sencillos que arrojan valores razonables en oportunidades de inversión complejas; 3) administran activamente el proyecto; 4) permiten utilizar probabilidades de riesgo en la inversión; 5) introducen asimetrías dentro de la distribución en los valores de oportunidad de la inversión; 6) muestran de forma explícita los factores que afectan e intervienen en la valuación; 7) arrojan resultado consistentes en condiciones reales de operación; y 8) dentro de la valuación se permite la incorporación del tiempo para estimar los posibles rendimientos en un horizonte de tiempo dado. 
En el caso de las nuevas tecnologías la técnica de opciones reales reflejó flujos de efectivo positivos desde el tercer año, sin importar que estructura de tasas se utilice; es decir, la técnica ante diversos escenarios de tasas de interés es más robusta. En tanto, el método de valor presente neto muestra flujos de efectivo positivos hasta el séptimo año, por lo que al tener menos periodos con ingresos podría existir la probabilidad creciente de rechazar el proyecto, situación que no ocurre con la opción real porque ésta internaliza la flexibilidad de las opciones de negocios que también tienen un valor y que la herramienta tradicional no considera y, por tanto, las técnicas pueden complementarse fácilmente y sería lo deseable en la toma de decisiones de un administrador de proyectos y de presupuestos de capital.

La flexibilidad u opcionalidad de tomar en un futuro una nueva decisión de inversión posee un valor presente $c$; es decir $\overline{V P N}=V P N+c$, entonces, aunque el valor presente neto sea negativo $\mathrm{VPN}<0$, si $\overline{V P N}=V P N+c>0$, el proyecto tiene la viabilidad de extender o implementar una nueva estrategia.

\section{Referencias}

Abetti, A. (1989). "Linking technology and business strategy", The Presidents Association, Frank J. Fabozzi. Greenwich, Advances in futures and options research 4, CT: JAI Press.

Bachini, D., J. Fronti y E. Márquez (2007). Valuación de un proyecto de inversión utilizando opciones reales borrosas. Facultad de Ciencias Económicas-Universidad de Buenos Aires: Centro de Investigación en Métodos Cuantitativos Aplicados a la Economía y la Gestión.

Black, F. y M. Scholes (1973). The pricing of options and corporate liabilities. Journal of Political Economy 81 (3): 637-654.

Brealey, R. y S. Myers (1991). Principles of corporate finance. 4a. ed., New York: McGraw-Hill.

Brennan, J. y L. Trigeorgis (1999). Project flexibility, agency and competition. New developments in the theory and application of real options. New York: Oxford University Press. 
Chatterjee, S., R. Wiseman, A. Fiegenbaum y C. Devers (2003). Integrating behavioral and economic concepts of risk into strategic management: The Taiwan shall meet. Long Range Planning (36): 61-79.

Cox, J., J. Ingersoll y S. Ross (1985). A theory of the term structure of interest rates. Econometrica 53 (2): 385-407.

Dixit, A. y R. Pindyck (2004). Investment Under Uncertainty. Princeton: Princeton University Press.

Duku-Kaakyire, Nanang D. (2004). Applications of real options theory investment analysis. Forest Policy and Economics 6: 539-552.

Doraszelski, U. (2001). The net present value method versus the option value of waiting: a note on Farzin, Huisman and Kort (1998). Journal of Economic Dynamics \& Control (25): 1109-1115.

George, M. y G.Jones (2006). Contemporary management: creating value in organizations, New York: McGraw Hill.

Holt, R. (2003). Investment and dividends under irreversibility and financial constraints. Journal of Economics Dynamics \& Control (27): 467-502.

Lander, D. y G. Pinches (1998). Challenges to the practical implementation of modeling and valuing real options. The Quarterly Review of Economics and Finance 38 (Special Issue): 537-567.

MacDougall, S. y R. Pike (2003). Consider your options: changes to strategic value during implementation of advanced manufacturing technology. The International Journal of Management Science (31): 1-15.

Meyers, S. (1977). Determinants of corporate borrowing. Journal of Financial Economics 5 (2): 147-175.

Miller, K. y H. Waller (2003), Scenarios, real options, and integrated risk management. Long Range Planning (36): 93-107. 
Moenaert, R. y W. Souder (1990), An information transfer model for integrating marketing an R\&D personnel in new product development projects. J. Prod Innov Manag.

Pennings, E. y O. Lint (1997). The option value of advanced R\&D. European Journal of Operational Research 103: 83-94.

Perlitz, Peske y Shrank (1999). Real options valuations: the new frontier in R\&D project valuation. $R \& D$ Management 29 (3): 255-269.

Pinches, G. (1998). Real options: developments and applications, Trustees of the University of Illinois. The Quarterly Review of Economics and Finance 38 (Special Issue): 533-535.

Sarmiento, J., E. Cayón y J. Sierra (2005). Opciones reales para las decisiones de inversión: Aspectos introductorios, técnicas modernas de gestión financiera. Colombia: Fundación Cultural Javeriana de Artes Gráficas 1: 25-71.

Tamayo, I. (2006). Flexibilidad estratégica y opciones reales en los procesos de cambio estratégico, tesis doctoral, Facultad de Ciencias Económicas y Empresariales, Departamento de Organización de Empresas, Universidad de Granada.

Trigeorgis, L. (1991). A log-transformed binomial numerical analysis method for valuing complex real options, Journal of Financial and Quantitative Analysis 26 (3): 309-326.

(1993). Real options and interactions with financial flexibility. Financial Management 22 (3): 202-224.

Valencia, H. y E. Gándara (2009). Relación entre incertidumbre e inversión en México, enfoque de opciones reales. Journal of Management, Finance and Economics 3 (2): 74-90.

Vasicek, O. (1977). An equilibrium characterization of the term. Journal of Financial Economics 5 (2): 177-188. 
Venegas-Martínez, F. (2006). Riesgos financieros y económicos: Productos derivados y decisiones económicas bajo incertidumbre, México: Internacional Thomson Editores.

Von-Braun y C. Friedrich (1991). The acceleration trap in the real word. Sloan Management Review Summer: 43-52. 
